\title{
Status of XMASS Experiment
}

\section{Shigetaka Moriyama ${ }^{1}$ for the XMASS collaboration}

Kamioka Observatory, Institute for Cosmic Ray Research, The University of Tokyo

Higashi Mozumi, Kamioka cho, Hida city, Gofu Prefecture, Japan

E-mail: moriyama@icrr.u-tokyo.ac.jp

The first phase of the XMASS experiment uses $800 \mathrm{~kg}$ of liquid xenon. In this study, structure of the detector and efforts for reducing background are discussed. Sensitivity of the detector for a cross-section of spin-independent interactions is expected to be $2 \times 10^{-45} \mathrm{~cm}^{2}$ for $50-100 \mathrm{GeV}$ weakly interacting massive particles (WIMPs) with one year of exposure. Detector construction in the Kamioka Observatory is underway and will be completed by September 2010.

Identification of Dark Matter 2010 (IDM2010)

Montpellier, France

July 26-30, 2010

\footnotetext{
$1 \quad$ Speaker
} 


\section{Introduction}

The XMASS experiment is a large-scale, multipurpose, low-background detector for research in astroparticle physics [1]. Liquid xenon was chosen as the target material for the detector because it has large photon yield and high atomic number as discussed in the references. As the first step, we decided to build a detector with $800 \mathrm{~kg}$ of single-phase liquid xenon, aiming to detect dark matter in the universe with a sensitivity of $2 \times 10^{-45} \mathrm{~cm}^{2}$ for the spinindependent interactions. Construction of the detector in the Kamioka mine was funded and started in April 2007. In this paper, we discuss the details of the detector structure, evaluated results of background, construction status, and the schedule after completion of the construction.

\section{Structure of $800 \mathrm{~kg}$ detector}

The detector is based on single-phase liquid xenon. We chose a spherical-shaped detector to maximize the detector uniformity and the volume-to-surface ratio. The latter condition was important because gamma rays are expected to be absorbed at the outer edge of the detector. This is the self-shielding effect shown in Fig. 1. On the other hand, weakly interacting particles such as dark matter particles can give uniform vertices distributions over the detector. By extracting events that occur only deep inside the detector, a sensitive search for dark matter can be conducted. Vertices of observed events are reconstructed using photo electron distribution detected by photo multiplier tubes (PMTs) placed on the surface of the sphere.

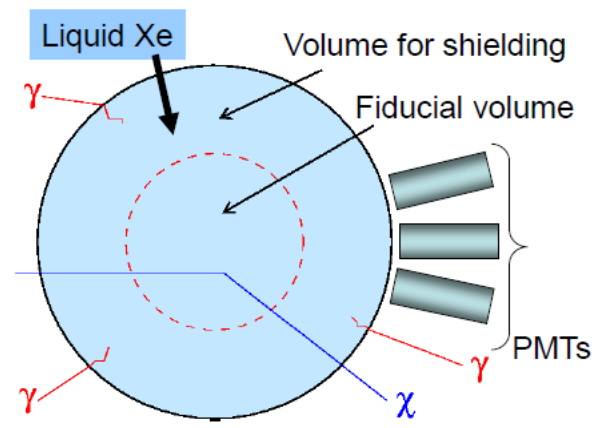

Figure 1 Reduction of radiations by self-shielding effect and uniform interactions by dark matter particles.

Placing PMTs on a spherical surface is an important process, and Fig. 2 shows the detailed structure of the detector. The PMTs have a hexagonal shape at the photocathode and are useful for covering more than $62 \%$ of the detector's inner surface. Their quantum efficiency around the scintillation wavelength of liquid xenon is high (more than $28 \%$ at $175 \mathrm{~nm}$ ). A triangular module consists of about ten PMTs. By combining 60 triangles, a pentakis dodecahedron can be constructed. The entire structure is immersed in a liquid xenon-filled chamber (Fig. 2). A light yield of more than 4.4 p.e./keV and an energy threshold at $5 \mathrm{keV}$ are expected with this setup. 


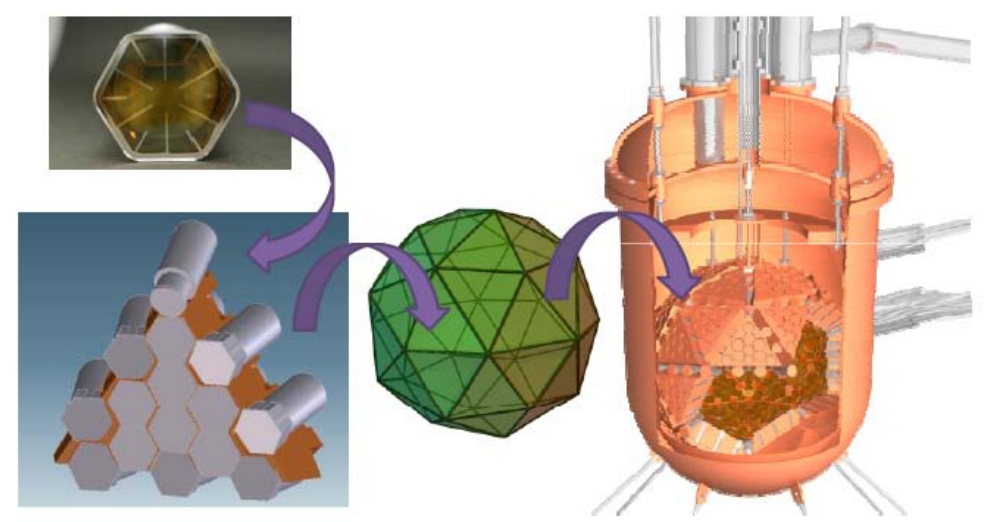

Figure 2 Structure of $800 \mathrm{~kg}$ detector.

\section{Efforts for background reduction}

For sensitivity of $2 \times 10^{-45} \mathrm{~cm}^{2}$ for the spin-independent cross-section, the required background above the energy threshold was $1 \times 10^{-4} \mathrm{keV}^{-1} \mathrm{day}^{-1} \mathrm{~kg}^{-1}$. The following steps were taken for achieving low background:

1. Reduction of radioactive contamination in the parts used for detector construction.

2. Construction of a large water active shield as the first application for dark matter detectors.

3. Development of a distillation system of xenon for reducing $\mathrm{Kr}$. The following subsections describe these efforts.

\subsection{Reduction of radioactive contamination}

The main concern in terms of radioactive contamination is related to the PMTs. We developed PMTs (R10789-11) with Hamamatsu Co. with special attention to radioactive contamination in their parts. Our goal was to reduce the radioactive contamination by one-tenth compared with that in the previous version of PMTs (R8778) developed for our prototype detector. The reduction was successful and Table 1 shows the measured radioactive contamination of a PMT.

Another material that may significantly contribute to the background is the copper material used for the PMT holder and the inner and outer chambers of the detector (Fig. 2). The problem is not only the level of radioactivity in the uranium and thorium chains but also the level of radioactivity by cosmic rays. Since the cosmogenic production rate of ${ }^{60} \mathrm{Co}$ in copper is around $2 \mathrm{mBq} / \mathrm{kg}$ [2], copper material was brought into the Kamioka mine within one month of electrorefining by Mitsubishi Materials Co.

Any other parts and materials that need to be used in the detector were measured by high purity germanium (HPGe) detectors. More than 250 potential candidates for parts were measured and selected on the basis of their contaminations. 
Figure 3 shows the simulated background spectra caused by gamma rays originating from the contamination of uranium chains in parts of the detector. Four different colored histograms show the background after various selections of reconstructed vertices. By repeating this analysis for other radioactive nuclei (see Table 1), it was shown that the total expected background in a $100 \mathrm{~kg}$ fiducial volume (indicated as " $20 \mathrm{~cm}$ shield" in the figure) was better than $1 \times 10^{-4} \mathrm{keV}^{-1} \mathrm{day}^{-1} \mathrm{~kg}^{-1}$.

\begin{tabular}{|l|l|}
\hline Radioactive nuclei & $\begin{array}{l}\text { Radioactivity in } \mathrm{mBq} \\
\text { with base parts }\end{array}$ \\
\hline U chain & $0.70 \pm 0.28$ \\
\hline Th chain & $1.5 \pm 0.31$ \\
\hline${ }^{40} \mathrm{~K}$ & $<5.1$ \\
\hline${ }^{60} \mathrm{Co}$ & $2.9 \pm 0.16$ \\
\hline
\end{tabular}

Table 1 Radioactive contamination of hexagonal PMT measured by germanium detectors. Numbers for $\mathrm{U}$ chain and $\mathrm{Th}$ chain were derived by assuming radioactive equilibrium.

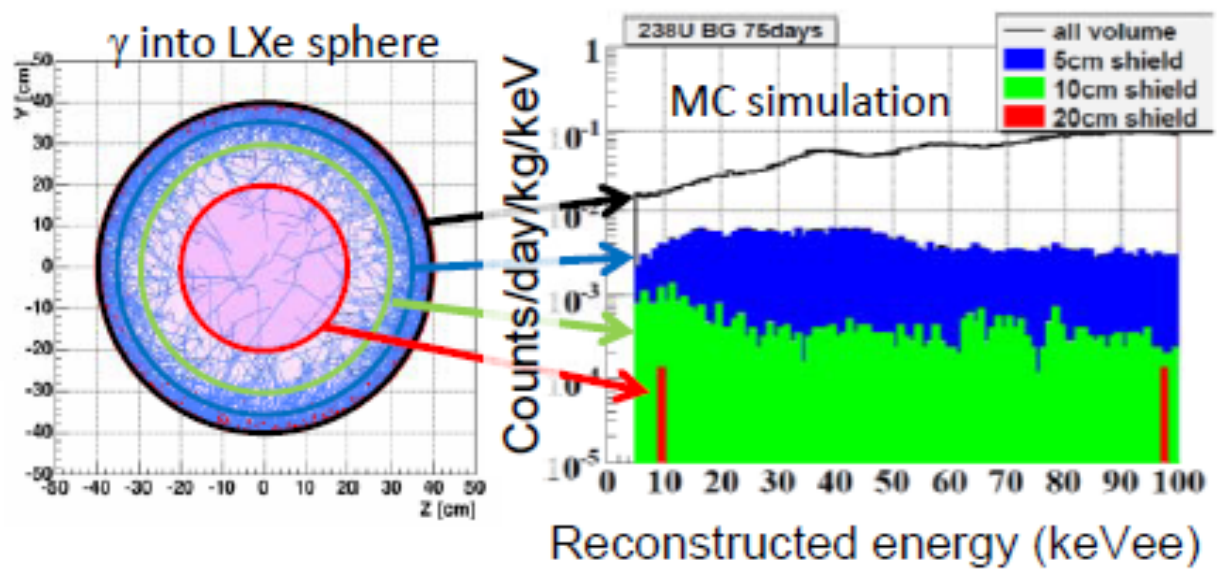

Figure 3 Energy spectra of background events after several fiducial cuts based on reconstructed vertices.

\subsection{Construction of larger water shield}

A pure water shield is better than a conventional lead and copper shield because of lesser production of neutrons by cosmic rays. Water is also useful for reducing the energy of incoming fast neutrons. By putting PMTs in the tank, the water also works as an active shield of muons. The water shield is $11 \mathrm{~m}$ high and $10 \mathrm{~m}$ in diameter and is equipped with 72 twenty-inch PMTs, providing a water shield that is more than $4 \mathrm{~m}$ thick for the $800 \mathrm{~kg}$ detector (Fig. 4). Gamma rays and neutrons from rock outside the detector were evaluated and shown to have a negligible effect (Fig. 5). Since the required minimum thickness of the shield is $2 \mathrm{~m}$, the water shield is useful for future extensions of the detector. 
The water purity can be kept very high using a water circulation system. We have an electronics hut on top of the tank; at the side of the tank, a cooling tower is installed for liquefaction and recondensation of xenon gas.

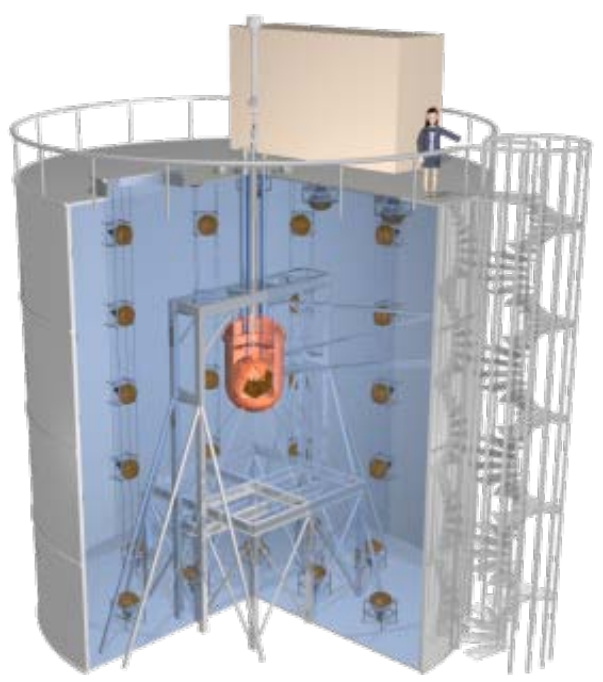

Figure 4 Active water shield for $800 \mathrm{~kg}$ detector.
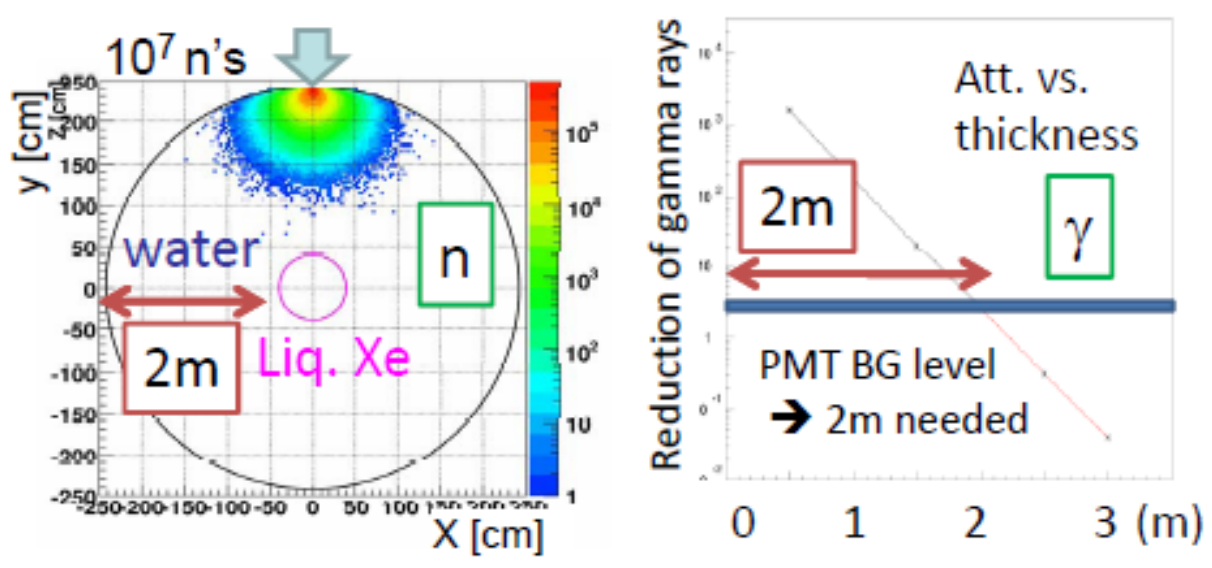

Figure 5 Shielding effect of water for neutrons and gamma rays.

\subsection{Development of distillation system of xenon for reducing $\mathrm{Kr}$}

${ }^{85} \mathrm{Kr}$ is a major source of radioactive background inside the detector. Depending on the source of xenon gas, Kr contamination in commercial xenon is $0.1 \mathrm{ppm}$ to a few ppm and must be reduced to 1 ppt. For that purpose, we developed a distillation tower based on our experience of a prototype system [3]. The distillation tower was designed to process a ton of xenon within 10 days, at an efficiency of more than $99 \%$. We are planning to process all of our xenon before putting it into the detector. 


\section{Construction status and schedule}

Just before the workshop, the inner chamber of liquid xenon was delivered. During August and September, the detector installation in the water tank is expected to be completed. Next, distillation of xenon and introduction of xenon into the detector should be completed. The data taking for dark matter search can be started depending on contaminations in liquid xenon.

Figure 6 shows the expected sensitivity with one year of exposure. It is expected to significantly improve the current sensitivity.

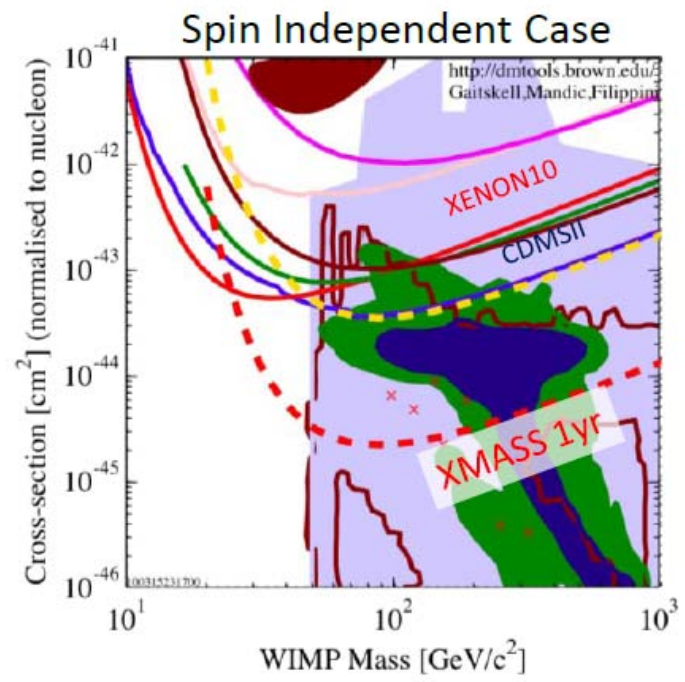

Figure 6 Expected sensitivity of XMASS 800 kg detector.

\section{Conclusion}

The $800 \mathrm{~kg}$ detector of liquid xenon is presently under construction. It is planned to be completed by September 2010. The expected sensitivity with one year of exposure is $2 \times 10^{-45}$ $\mathrm{cm}^{2}$ for the spin-independent cross-section with background $1 \times 10^{-4} \mathrm{keV}^{-1} \mathrm{day}^{-1} \mathrm{~kg}^{-1}$ in the fiducial volume of $100 \mathrm{~kg}$. Data taking for dark matter search is expected to start soon.

\section{References}

[1] Y. Suzuki, hep-ph/0008296

[2] G. Heusser, M. Laubenstein, H. Neder, Proc. of Intern. Conf. Isotop. Environm. Studies Aquatic Forum, Monaco, Oct. 25, 2004

[3] K. Abe et al. (the XMASS collaboration), Astroparticle Physics, 31 (2009) 290. 